\title{
Surgical management of pericarditis in Zaria, Nigeria
}

\author{
OA MABOGUNJE, CO ADESANYA, MS KHWAJA, JH LAWRIE, \\ GM EDINGTON
}

From the Departments of Surgery, Medicine, and Pathology, Ahmadu Bello University, Ahmadu Bello University Hospital, Zaria, Nigeria

ABSTRACT Over eight years, 58 rural Nigerians with pericarditis were treated surgically in Zaria using basic surgical facilities. Eighteen patients had purulent pericarditis, associated with staphy- in lococcal pneumonia in children, or pneumococcal pneumonia in adults. Treatment with antibiotics and prompt pericardiectomy appeared to be superior to drainage, since a quarter of those initially treated with surgical drainage developed early constriction and required pericardiectomy soon after. Thirteen patients had chronic pericardial effusions, of whom one had epicardial constriction and two had cardiomyopathy. Twenty-seven patients had chronic constrictive pericarditis but $\frac{c}{5}$ tuberculosis was confirmed histologically in three only. Echocardiographic findings remained unchanged in five patients evaluated before and after pericardiectomy. Eight of the 13 patients who died had already developed myocardial or hepatic insufficiency before operation, because of late presentation or diagnosis. Greater awareness of the significance of precordial pain in this rural population where ischaemic heart disease is rare would help in making an earlier diagnosis.

Bacterial infections are an important cause of pericarditis in tropical Africa. Purulent pericarditis often presents in a life-threatening manner demanding urgent attention especially in children and adolescents. ${ }^{1}$ Chronic pericarditis, on the other hand, is more insidious in its mode of presentation, often having no relationship to any previous acute episode, frequently presenting with misleading, predominantly abdominal signs, and it had not apparently been treated surgically in this region before 1971. Surgery for these conditions is, however, well within the scope of basic surgical facilities, even when sophisticated thoracic equipment is entirely lacking. We present our experience in the management of pericarditis over eight years at the Ahmadu Bello University Hospital in Zaria, serving a predominantly rural population. Cardiac catheterisation was not available but during 1977 and 1978 most of the patients had echocardiography before and after surgery. ${ }^{2}$

\section{Patients}

From 1971 to 1978,58 patients with pericarditis were operated upon. They have been divided into three groups: purulent pericarditis (18), chronic pericardial effusion (13), and chronic constrictive pericarditis (27).

Address for reprint requests: Dr OA Mabogunje, ABU Hospital, Zaria, Nigeria.

\section{Results}

The various surgical procedures and the post- $\frac{3}{3}$ operative deaths in the three groups of patients are summarised in the table.

Table Summary of operations and deaths (in parentheses)

\begin{tabular}{lccl}
\hline Procedure & $\begin{array}{l}\text { Purulent } \\
\text { pericarditis pericardial } \\
\text { effusion }\end{array}$ & $\begin{array}{l}\text { Chronic } \\
\text { constrictive } \\
\text { pericarditis }\end{array}$ \\
\hline $\begin{array}{l}\text { Pericardiocentesis } \\
\text { Tube pericardiostomy }\end{array}$ & $2(1)$ & 2 & - \\
$\begin{array}{l}\text { Pericardial window } \\
\text { Primary pericardiectomy }\end{array}$ & $9(1)$ & 1 & - \\
$\begin{array}{l}\text { Secondary pericardiectomy } \\
\quad \text { (initially had pericardiostomy) }\end{array}$ & 2 & $7(3)$ & - \\
Total & $18(4)$ & $13(4)$ & $27(5)$ \\
\hline
\end{tabular}

PURULENT PERICARDITIS

Of the 18 patients in this group, 13 were males andeo five females, eight being younger than 19 years. Most $\frac{C}{\mathscr{C}}$ of these patients had a short duration of symptoms (median seven days) and only three had longer $T$ durations, of six to 12 months. Thirteen were $\stackrel{\vec{\Phi}}{\bar{D}}$ admitted with an initial diagnosis of pneumonia, $\stackrel{\Phi}{\varrho}$ which in one child was a complication of measles. $\stackrel{\unrhd}{\unrhd}$ Four had large pleural effusions and one had tropical $\sigma$ pyomyositis with multiple abscesses in the right $O$ thigh, left shoulder, and left foot. Productive cough응 
with purulent or blood-stained sputum was the most common symptom in 17 patients $(94 \%)$, fever ranging up to $40^{\circ} \mathrm{C}$ in $15(83 \%)$, dyspnoea in 12 , and orthopnoea in six. Precordial pain and epigastric pain were present in six patients each and eight complained of weakness. Fourteen patients $(78 \%)$ were toxic or delirious. An elevated jugular venous pressure (JVP) was found in 15, hepatomegaly in 14, a quiet heart with an impalpable apex in eight together with basal crepitations, decreased breath sounds and dullness in the lung fields, leg oedema in eight and ascites in six. A pericardial friction rub was heard in a few patients only.

Pulmonary consolidation or infiltration or pleural effusion was shown radiologically in all, but an enlarged globular heart was initially seen in only half the cases. The principal finding on the ECG obtained in nine cases was sinus tachycardia with a flat or inverted $\mathrm{T}$ wave in the chest leads. Blood counts showed a leucocytosis ranging from 11000 to 36000 in half the cases, with neutrophilia and toxic granulation. Gram stain of the sputum smear showed leucocytes and bacteria in many of those who had pneumonia.

The diagnosis was confirmed by pericardiocentesis. From the pus aspirated, $S$ pneumoniae was cultured in three, $S$ aureus in three, and $H$ influenzae in one. There was no growth in six instances but $S$ pneumoniae was cultured from the sputa of four patients. In one patient with pneumococcal pneumonia who died, S typhi was cultured from the blood. The Ziehl-Neelsen (ZN) stain of multiple smears of sputa from five patients, pericardial pus from three and the pleural fluid in one, showed no acid-fast bacilli.

All patients were initially treated with parenteral penicillin and streptomycin, varied later according to the results of sensitivity tests. Nine patients were managed by tube pericardiostomy using a trocar and cannula through the subxiphoid approach; six of these improved, two developed pericardial constriction and required pericardiectomy before improving and one $(11 \%)$ died of $S$ typhi septicaemia two days after operation. Primary pericardiectomy was performed in seven cases, five of whom improved. Two died; a 16-year-old boy who had been treated for tuberculosis for six months before operation died of pulmonary oedema one month later and a 22-yearold man who had loculated empyemas died of staphylococcal septicaemia three weeks after operation.

The child with tropical pyomyositis improved after needle aspiration of the pericardial collection and incision and drainage of her multiple skeletal abscesses. The parents of an 8-year-old boy refused consent for operation and removed him from the hospital after a few needle aspirations. He was returned moribund to the hospital a month later but died a few minutes after arrival.

Overall four patients died in this group but excluding the boy for whom operation was refused, the mortality was three out of $17(18 \%)$, two out of nine $(22 \%)$ for those who had pericardiectomy, and one of nine $(11 \%)$ for those who had tube pericardiostomy.

\section{CHRONIC PERICARDIAL EFFUSION}

Twelve men and one woman ranging in age from 20 to 60 years were operated upon for chronic pericardial effusion. The duration of symptoms ranged widely from one month to four years, with a median of five months.

The major symptoms were precordial or epigastric pain in all, dyspnoea in 10 patients, and variously a cough, leg swelling, weight loss, abdominal swelling, and palpitations. On examination, an elevated jugular venous pressure and a quiet heart with an impalpable apex and hepatomegaly were present in 10. Seven patients had ascites, in one actually leaking through a traumatic abdominal puncture. The pulse was of small volume in all, and a paradoxical pulse, indicating chronic tamponade, was found in nine.

An enlarged globular heart shadow with clear lung fields (fig 1) was the prominent finding on the chest radiographs, and three patients also had a pleural effusion. An ECG was obtained in 10 patients. Sinus rhythm was present in eight, two of whom had ectopic ventricular beats and one electrical alternans; one patient had sinus tachycardia and the other showed atrial fibrillation. There was a low QRS voltage in all cases with a flat or depressed $T$ wave in the limb and chest leads.

Only two patients were anaemic. However, the

Fig 1 Chest radiograph of a patient with a large pericardial effusion, showing the large globular cardiac shape. 
blood urea nitrogen was elevated in six patients, four of whom eventually died. Only one of these had a large haemorrhagic effusion, possibly of uraemic origin.

Diagnosis was confirmed by pericardiocentesis, a clear straw-coloured to brownish fluid being obtained in all but one instance. The volume ranged from 700 to $3000 \mathrm{ml}$. In about half the patients, no organism was recovered from the pericardial fluid, blood, sputum, or pleural fluid. The $\mathrm{ZN}$ stain was performed on several sputum smears from five of the patients but acid-fast bacilli were not seen.

In general, the patients were treated with diuretics and anti-tuberculous drugs. Half of them had also been treated with digoxin by their referring physicians; three had received steroids. Two patients improved after pericardiocentesis and refused further surgery but remained on medications. A third patient also improved after tube pericardiostomy. A pericardial window was created in seven patients, four of whom improved. Three patients died-one from exsanguination when the right ventricle was lacerated while creating a pericardial window through the subxiphoid approach, one of myocardial failure, and the other of liver failure. Pericardiectomy was performed on three patients. Two improved and one died of myocardial failure. Thus the mortality after a pericardial window operation was three out of seven $(43 \%)$. The pericardium was relatively normal in the two patients in this group who died of myocardial failure. In retrospect they were both thought to have had a cardiomyopathy.

\section{CHRONIC CONSTRICTIVE PERICARDITIS}

Twenty-seven patients (17 male and 10 female) had pericardiectomy for chronic constrictive pericarditis. The youngest was an infant of 8 months. One was a child of 2 years who had had a pericardial window made three months previously in another hospital for purulent pericarditis. Six patients were in the second decade, eight in the third, seven in the fourth, and four were over 40 years of age. Symptoms had been present from two months to five years (median one year). Many of the patients had been treated for congestive heart failure or cirrhosis of the liver at outstation hospitals before referral to this hospital and two middle-aged patients were already jaundiced at the time of admission.

The major symptoms were dyspnoea, abdominal distension, cough, and leg swelling. Nine patients complained also of precordial pain, 12 of epigastric pain, six of palpitations, and one patient of syncopal attacks. All these patients had an elevated jugular venous pressure reaching the angle of the jaw, and obvious hepatomegaly. The apex beat was impalpable in two-thirds of the patients, and half of them had $\frac{\text { के }}{5}$ ascites and leg oedema. A pericardial knock and a $\frac{C}{0}$ friction rub were each recorded in three patients. $\frac{\overline{\bar{N}}}{\overline{0}}$ Most patients had a blood pressure in the low range $\widehat{\Phi}$ of normal and a small volume pulse. The pulse was irregular in four but only half of the patients hadis tachycardia.

The heart was usually of normal size on the chest $\overrightarrow{-}$ radiograph, and nine cases had pericardial calcifi- $\vec{\sigma}$ cation best seen in the lateral projection (fig 2). The

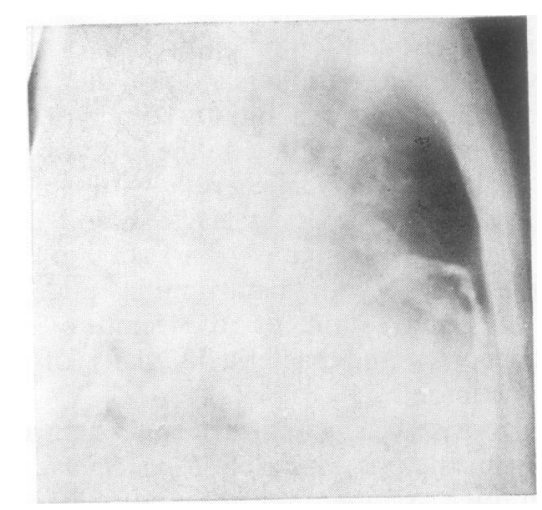

Fig 2 Lateral chest radiograph showing a thick ring of calcium, in a patient with calcific constrictive pericarditis.

right upper mediastinum was widened because of the distended superior vena cava. The lung fields wereo usually clear. However, in six cases the heart shadow. was enlarged giving the impression of a left ventric? ular aneurysm in one instance. Five cases had pleuraB effusions and two pleural thickening.

The ECG obtained in 16 patients showed sinus rhythm in nine, atrial fibrillation in four, sinus? tachycardia in two, and incomplete right bundle branch block in one. There was low QRS voltage with flat or inverted $T$ waves in the limb and pre ${ }_{0}^{N}$ cordial leads.

The blood urea nitrogen (BUN), electrolytes, and liver function tests were within normal limits except in the two jaundiced patients. One of these had के BUN of $30 \mathrm{mmol} / \mathrm{l}$ and total bilirubin of $373 \mathrm{mmol} / 1 \stackrel{\circ}{+}$ They both had fetor hepaticus on admission but the hepatitis-associated antigen was not present in theip sera. Acid-fast bacilli were not seen on the $\mathrm{ZN}$ stain of several sputum smears but the sputum culture irg two patients each grew $S$ pneumoniae and Klebsiellos spp. At thoracotomy, two patients had thick loculated pericardial abscesses which were sterile of 
culture. Seven of these patients had been on triple anti-tuberculous therapy on the suspicion of tuberculous pericarditis.

Under endotracheal anaesthesia, left anterolateral thoracotomy was performed through the bed of the fifth or sixth rib. In all, the constricting pericardium was much thickened and adherent to the myocardium, the pericardial space being completely obliterated in all but four. In one of these there was loculation of $300 \mathrm{ml}$ of clear fluid; in a second, there was a large blood clot overlying and constricting the right ventricle; in the third, thick yellow pus was encased over the right ventricle; and in a fourth, a horse-shoe shaped cavity full of pus was encased over the right and left ventricles and had simulated a ventricular aneurysm on the chest radiograph. By sharp and blunt dissection, a plane of cleavage was developed deep to the epicardium and both pericardial layers were excised from posterior to the left phrenic nerve to as far to the right under the sternum as was feasible. An attempt was made to free most of the anterior surfaces of the left and right ventricles, the left atrioventricular groove and the ventricular apex. Patches of calcified or densely adherent epicardium were left, bone shears being used occasionally. The chest was closed with an underwater seal.

Resuscitation was not successful in two patients who suffered cardiac arrest on the operating table immediately after pericardiectomy and in a third in the recovery area. The two jaundiced patients died of progressive liver failure nine and 13 days respectively after surgery. Thus five $(19 \%)$ of the 27 patients in this group died.

The postoperative period was complicated in two patients by cholestatic jaundice which cleared over three weeks, in two others by empyema, and in one patient by a post-pericardiectomy syndrome.

Five patients with chronic constrictive pericarditis had echocardiographic evaluation before pericardiectomy. They all demonstrated the sharp abnormal posterior motion of the left ventricular posterior wall in early diastole and abnormal motion of the ventricular septum. When the patients were studied two to four weeks after operation, these abnormalities were unchanged but the posterior left ventricular wall motion was perhaps less sharp. In one of them, the much thickened pericardium was less prominent after surgery. Two other patients who had constrictive pericarditis were studied only after pericardiectomy. In one the motion of the left ventricular posterior wall was normal six weeks after operation, the patient having improved clinically; in the other, the abnormal posterior motion of the left ventricular posterior wall was still present one year after operation.
PATHOLOGICAL FINDINGS

In the first group of patients, those with purulent pericarditis, the histological appearance of the pericardial tissue was of non-specific chronic inflammation in four, acute pyogenic inflammation in three, and tuberculosis in two.

Eleven specimens were obtained from the patients with pericardial effusion, of which six showed nonspecific chronic inflammation, and three showed tuberculous changes. The remaining two were from the patients who were considered to have a cardiomyopathy and these were grossly normal with minimal fibrosis.

In the chronic constrictive pericarditis group, the excised pericardium varied from $5-20 \mathrm{~mm}$ in thickness. Only three specimens showed tuberculous changes. The other 24 showed non-specific chronic inflammation varying from fibrosis with sparse infiltration of round cells to dense collagenous tissue, with areas of dystrophic calcification in nine instances. In one case, the grossly "calcified nodules" were found histologically to be focal accumulations of cholesterol crystals. However, in the two youngest patients, foci of fibrinoid necrosis suggestive of a rheumatic origin were present.

The thickest pericardial specimens came from two patients aged 20 and 14 years respectively. The former had purulent pericarditis while the latter had chronic constrictive pericarditis. In both cases PASpositive helminth-like structures were present with a dense chronic inflammatory reaction around them. The exact role of these "helminths" in the pathogenesis of pericardial thickening is not clear.

\section{FOLLOW-UP}

Many of these patients came from long distances to this hospital and usually returned to their local hospitals one to three months after operation. Thus of the $\mathbf{4 5}$ patients discharged long-term information is available only in nine. None of those with purulent pericarditis were seen in our cardiac clinic beyond three months, when they were symptomatically improved. Of those operated on for pericardial effusion, two were seen one year later, one was still in atrial fibrillation and taking digoxin and diuretics for moderate ascites and the other was well, taking no drugs. One of the two with cardiomyopathy had not deteriorated. Six patients operated on for chronic constrictive pericarditis were followed for one to four years. Four were much improved, taking no drugs while two have reduced venous pressure and liver size but are still on digoxin and diuretics.

\section{Discussion}

Since the antibiotic era, purulent pericarditis appears 
to be less common in the Western world, but is still a major clinical concern in Africa. ${ }^{13}$ In children $S$ aureus is the most common bacterial cause, ${ }^{4-6}$ and this was found to be true also in Northern Nigeria. Two of the three patients with $S$ aureus pericarditis in this series were children. In one, purulent pericarditis occurred along with fulminating tropical pyomyositis, in which the multiple skeletal abscesses may divert the doctor's attention, the pericarditis being discovered later at necropsy. ${ }^{7}$ In adults $S$ pneumoniae was the most commonly implicated organism both in the pericardial pus and the sputa of those who had pneumonia, thus conforming with the pattern of pneumococcal pericarditis elsewhere. ${ }^{8}$ The patient with $H$ influenzae pericarditis was a 28 year-old woman who had pneumonia and bacteraemia. Although this organism is typically found in childhood pericarditis, two other adult cases similar to ours have been reported. ${ }^{9}$ There was no evidence for immune deficiency in any of our adult cases. Most of these patients presented with the predisposing infection and the complicating pericarditis on admission, and it was important to establish both diagnoses promptly and institute appropriate management. It is thus possible that not all the patients who have purulent pericarditis are recognised clinically. In Uganda for example, D'Arbela $e t$ $a l^{1}$ found a large number of unsuspected cases at necropsy.

From the large experience in East Africa, a scheme of management combining early pericardiectomy with the use of antibiotics has been recommended. ${ }^{110}$ The advantages include a more thorough drainage of the pericardium and the prevention of acute constriction which may follow limited drainage by tube or window. This happened to two adults initially treated by tube pericardiostomy in this hospital, and to a child initially treated by a pericardial window at another hospital three months before his admission here for constrictive pericarditis. For similar reasons other authors have also recommended this course. ${ }^{11} 12$ We have usually performed a subxiphoid pericardiostomy in the past but more recently have performed some pericardiectomies for this condition. Although the mortality of two $(22 \%)$ of the nine patients treated with pericardiectomy in this group is comparable with the results of others, 4810 it is not yet possible to recommend this course of action firmly for all cases of purulent pericarditis in view of the small numbers in this series.

The cause of chronic pericardial effusion in this region is not clear. The connective tissue diseases such as rheumatoid arthritis and lupus are rarely seen here, and none of the patients had received radiation therapy to the chest. In two patients from this series, large effusions were found in association with cardiomyopathy. In this group also was the 20 -year- $\frac{C}{0}$
old man who had a large effusion in association with $\frac{}{\bar{c}}$ a partially calcified and constricting epicardium. $\mathrm{He} \underset{\widetilde{\sigma}}{\overrightarrow{\mathrm{d}}}$ thus fits into the effusive-constrictive pattern out- $\underline{a}$ lined by Hancock, ${ }^{13}$ most of whose cases were idiopathic.

Tuberculosis is commonly presumed but infre- $-\overrightarrow{-}$ quently proved. In only three of the 27 cases of $\vec{\omega}$ constrictive pericarditis was there histological evidence of tuberculosis. Some of those in the non- $x$ specific chronic inflammatory group may well have had tuberculous or viral pericarditis initially, though 0 others may have resulted from inadequately treated \& purulent pericarditis. ${ }^{11415}$

After pericardiectomy through a left thoracotomy, $\stackrel{\circ}{?}$ the rate of clinical improvement has often been less $\vec{\longrightarrow}$ impressive than the dramatic improvement in cardiac action and filling seen at the time of operation. The $\frac{\sqrt{\omega}}{\mathrm{s}}$ postoperative echocardiogram in five of our patientsshowed that the abnormality in the motion of the left $\oplus_{\infty}$ ventricular septum persisted up to four weeks. This.agrees with the findings of Pool et al. ${ }^{16}$ The haemodynamic findings in similar patients from South Africa ${ }^{17} 18$ showed that right ventricular function remained unimproved with persistent elevation in the end-diastolic pressure. As with their patients and $\stackrel{\odot}{\Phi}$ others from Southern Nigeria, ${ }^{19}$ residual cardiac $\overrightarrow{\vec{P}}$ failure of variable severity required continuation of 3 drug treatment in some of our patients for weeks after operation. This has been ascribed to the inadequate pericardiectomy of the right heart afforded by the limited access of left thoracotomy, oro to myocardial dysfunction. Further clinical improve $\frac{0}{x}$ ment can occur over several months and the fouro patients in this series followed up to four years have demonstrated this pattern.

Pericardial disease represents 2 to $4 \%$ of cardiac disorders in this region 1420 but constitutes theo majority of those cardiac problems which can be managed by the limited surgical facilities available in a rural setting. Potentially great relief and occasiona permanent cure may be afforded this group of patients. It is thus regrettable that many patients with chronic pericardial effusion and chronic constrictive pericarditis presented late when irreversible myo-0 cardial or hepatic insufficiency had developed. In this series, five of the 13 deaths were due to myocardia failure presumably the result of fibrosis or myo cardial fibre atrophy, ${ }^{1521}$ and three patients died ofo liver failure within two weeks of pericardiectomy Since in this rural African population, ischaemi® heart disease is exceptionally rare, ${ }^{22} 23$ a history of precordial or mid-chest pain probably indicates peri carditis. ${ }^{24} \mathrm{~A}$ greater awareness of this association among doctors working in outstation hospital 
should prompt early referral for further assessment and management. We can then hope to see a decreased mortality from pericarditis and pericardiectomy in this region.

COA was supported by the National Institute for Medical Research (Grant MR/RG AD 136). We thank Mr Olajide of the Central Histopathology Laboratory for technical assistance and Mr Edwin Arii for secretarial help.

\section{References}

${ }^{1}$ D'Arbela PG, Patel AK, Grigg GL, Somers K. Pericarditis, with particular emphasis on pyogenic pericarditis: a Ugandan experience. East Afr Med J 1972;49: 803-16.

${ }^{2}$ Adesanya CO, Sanderson JE. M-mode echocardiography in the diagnosis of heart diseases in Africans. Trans $R$ Soc Trop Med Hyg 1979;73:400-5.

${ }^{3}$ Fowler MO, Manitsas GT. Infectious pericarditis. Prog Cardiovasc Dis 1973;16:323-36.

4 Weir EK, Joffe HS. Purulent pericarditis in children: an analysis of 28 cases. Thorax 1977;32:438-43.

5 Cameron EWJ. Surgical management of staphylococcal pericarditis. Thorax 1975;30:678-81.

${ }^{6}$ Okoroma EO, Perry LW, Scott LP. Acute bacterial pericarditis in children. Report of 25 cases. Am Heart J 1975; 90:709-13.

7 Thomas GE, Francis TI, Smith JA. Cardiac complications of tropical pyomyositis. Niger Med J 1974;4:29-31.

${ }^{8}$ Kauffman C, Watanakunakorn C, Phair JP. Purulent pneumococcal pericarditis. A continuing problem in the antibiotic era. Am J Med 1973;54:743-50.

${ }^{9}$ Crossley K, Bigos T, Joffe CD. Hemophilus influenzae pericarditis. A report of two cases in adults with a summary of the literature. Am Heart J 1973;85:246-51.
${ }^{10}$ Sinclair MC. Acute pyogenic pericarditis. The role of pericardiectomy. East Afr Med J 1978;55:136-42.

${ }^{11}$ Caird R, Conway N, McMillan IKR. Purulent pericarditis followed by early constriction in young children. Br Heart J 1973;35:201-3.

12 Thomas GI, Gregores BJ, Perry DU, Andrus W. Pericardiectomy for acute constricting staphylococcal pericarditis. Report of a case. $N$ Engl J Med 1962;267:440-2.

${ }^{13}$ Hancock EW. Subacute effusive-constrictive pericarditis. Circulation 1971;43:183-92.

${ }^{14}$ Ladipo GOA, Froude JRL, Parry EHO. Pattern of heart disease in adults of the Nigerian Savanna: a prospective clinical study. Afr J Med med Sci 1977;6:185-92.

15 Hirschmann JV. Pericardial constriction. Am Heart J 1978 96:110-22.

${ }^{16}$ Pool PE, Seagren SC, Abbasi AL, Charuzi Y, Kraus R. Echocardiographic manifestation of constrictive pericarditis: abnormal septal motion. Chest 1975;68:684-8

${ }^{17}$ Gotsman MS, LeRoux BT, Rogers NMA, Van der Horst RL, Winship WS. Immediate haemodynamic results of pericardiectomy. $S$ Afr Med J 1972;46:3-8.

18 Coleman AJ, Moyes DG, Wheatley DJ, Henderson BJ, Rogers NMA. Immediate effects of pericardiectomy $J$ Thorac Cardiovasc Surg 1973;66:803-6.

19 Grillo IA, Falase AO, Akinyemi OO, Basile U, Lagundoye SB. Calcific constrictive pericarditis. Report of four cases in Nigerians. Niger Med J 1979;9:135-9.

${ }^{20}$ Beet EA. Rheumatic heart disease in Northern Nigeria. Trans $R$ Soc Trop Med Hyg 1956;50:587-92.

21 Dayem MKA, Wasfi FM, Bentall HH, Goodwin JF, Cleland WP. Investigation and treatment of constrictive pericarditis. Thorax 1967;22:242-52.

${ }^{22}$ Adesanya CO, Nirodi NS. Fatal coronary atherosclerotic heart disease in a Nigerian. Case report with necropsy findings. J Trop Med Hyg 1977;80:219-23.

${ }^{23}$ Falase AO, Cole TO, Osuntokun BO. Myocardial infarction in Nigerians. Trop Geogr Med 1973;25:147-50.

${ }^{24}$ Schrire V. Experience with pericarditis at Groote Schuur Hospital, Cape Town. An analysis of 160 cases studied over a six-year period. $S$ Afr Med J 1959;33:810-7. 